
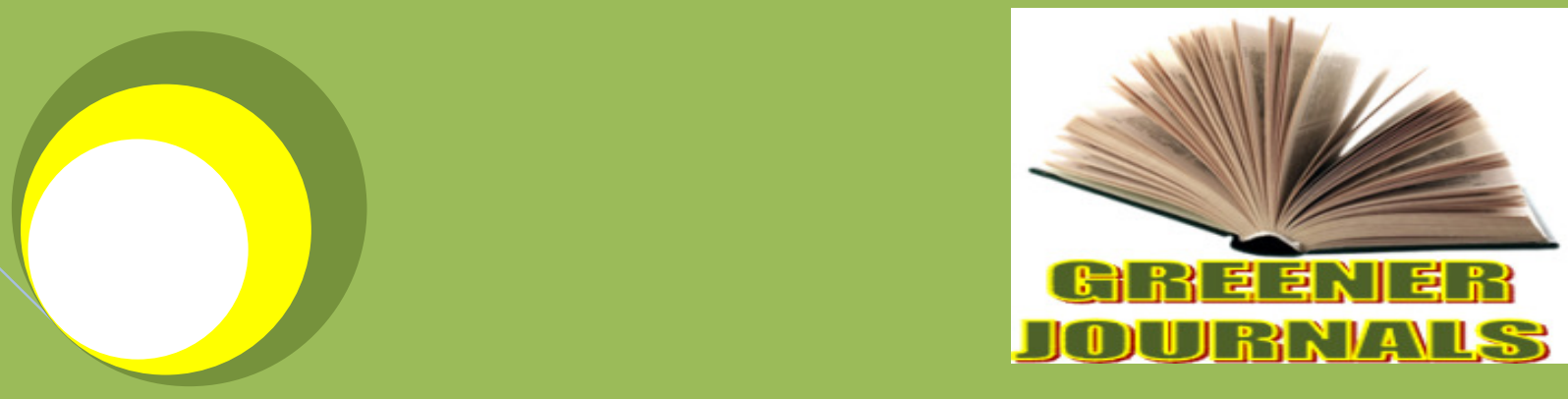

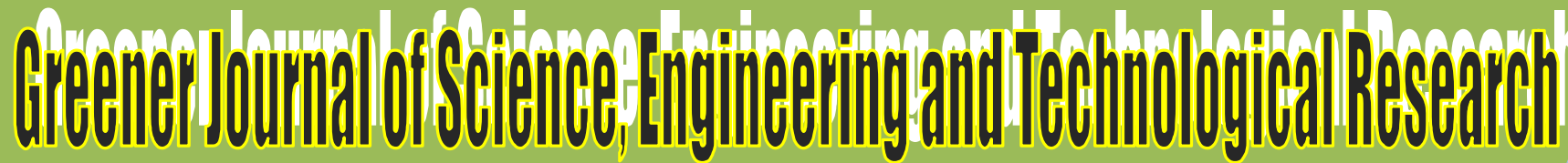
ISSN: 2276-7835 Impact Factor 2012 (UJRI): 0.7563 ICV 2012: 5.62

Electron Interaction at Low Filling in Two Dimensions Using a Simplification of the Modified Lanczos Technique

By

E.O. Osafile

D.O. Ojuh

J.O.A. Idiodi 


\title{
Electron Interaction at Low Filling in Two Dimensions Using a Simplification of the Modified Lanczos Technique
}

\author{
E.O. Osafile ${ }^{1^{*}}$, D.O. Ojuh ${ }^{2}$ and J.O.A. Idiodi ${ }^{3}$ \\ ${ }^{1}$ Department of Physics, Federal University of Petroleum Resources, Effurun, Delta State. \\ ${ }^{2}$ Department of Basic Sciences, Benson Idahosa University, Benin city, Edo State. \\ ${ }^{3}$ Department of Physics, University of Benin, Benin City. \\ ${ }^{* 1}$ Corresponding Author's E-mail: eromopower@yahoo.com
}

\begin{abstract}
In this paper, a detailed study of the dynamics of the behavior of two electrons in two dimensions is carried out. Over the years, the Hubbard model has been heavily used as one of the standard models for the study of strongly correlated electron systems. Despite this, the mechanism of interaction of these electrons in two- dimensional systems is yet to be properly understood.

Based on this clarity problem, a modification of the standard Lanczos algorithm which is slightly different, simpler and more analytical than that presented by Dagotto in his review paper is used in a concerted attempt to produce relevant results for the correlated ground state energies and wave functions and the behavior of the interacting pairs is explained with the aid of the well known single band Hubbard model. Hence this study has been able to develop, stabilize and utilize a simplified modification of the standard Lanczos technique.

From results obtained, for large and positive values of $u$, interaction is repulsive while it is attractive for negative values of $u$. The wave function can be readily obtained at the end of each step of iteration.
\end{abstract}

Keywords: Standard Lanczos technique, Hubbard model, Modified Lanczos technique, two- dimensional systems.

\section{INTRODUCTION}

There is a need to understand the anti-ferromagnetic properties in the Hubbard model of strongly correlated electron systems. In the Hubbard model and related models, one important parameter is the filling. Much work has been devoted to the high density case of near half filling since it is believed to be relevant for high temperature superconductivity (Lieb, 1995), but the low filling regime is also of interest; for example, it plays an important role in theoretical studies of the breakdown of the Fermi liquid theory in two dimensions. This work is limited to the case of two electrons in two- dimensional lattices specifically $3 \times 3$ and $5 \times 5$ square lattices. This is because a proper understanding of the procedure for obtaining the ground state energy and wave functions will lay a good foundation for the calculation of other low energy ground state properties of the interaction of higher number of electrons.

Having made a choice of which model to work with, the method or technique to study this model effectively becomes another challenge. If this is not properly chosen, it limits the extent of the work and the accuracy of results obtained. To check this, great effort was made in the review work of Dagotto (1994) to present several techniques that has been applied in the extensive study of highly correlated electron systems using the Hubbard model. One of the problems encountered was that there were no well controlled analytical techniques to analyze them in two dimensions. According to Dagotto's review paper, two methods were found to be self consistent; these were the mean free theories and the variation approximations, but there were no standard ways to judge if they actually describe the properties of the ground state or if they converge to excited states. Of these aforementioned techniques, the exact method provide the exact solution, however, because of the exponential growth of the Hilbert space with the lattice size, exact calculation becomes inadequate to handle relatively large clusters. Even if some extrapolations were done, small size systems are inadequate in describing the phase transition region in the macroscopic limit. This fueled the need to look for an adequate approximation method that can produce near accurate results.

It is in the light of this that a simple modification of the Lanczos algorithm was developed to extract the desired information from the system operated on by the Hamiltonian. Hence for this research paper, a simplified 
modification of the Lanczos technique is employed. The method developed in this work reveals a clear cut analytical formula for obtaining the improved ground state energy and wave function by every step of iteration taken. Values can be inputted manually into the formula and results obtained without heavy numerical involvement.

In the modified Lanczos technique presented by Dagotto, two higher powers of $\langle\mathrm{H}\rangle$ that is $\left\langle\mathrm{H}^{2}\right\rangle$ and $<\mathrm{H}^{3}>$ must be obtained before the iteration to obtain the next improved ground state energy and wave function can be obtained. However in the method presented here, just one power of $\langle\mathrm{H}\rangle$, that is $\left\langle\mathrm{H}^{2}\right\rangle$ is needed for the next iteration to be carried out. Result obtained from the iterations shows that there is easy access to obtaining some of the dynamical ground state properties like the correlation functions. Hence the aim of this research is to investigate to a high level of accuracy, the low energy properties like the ground state energies and wave functions of the various electron interactions in the strongly correlated electron system at $u=0$, in the large $(u>0)$ and small $(u<0)$ limit.

Generally, the plan of this work is as follows, in section 1, the simplified modification of the Lanczos technique is developed. Furthermore, the method is applied to finite sized lattices in two dimensions for two electrons. The results obtained from the applications done in section I is presented in section II using tables. Interpretation and discussion of these results is carried out in section III. Comparison is also made with previous results obtained using other techniques. In section IV the study is concluded.

\section{METHODOLOGY}

In recent years, the Hubbard model has received increasing attention for its relevance for high- $T_{c}$ superconductivity, quantum anti-ferromagnetism, and ferromagnetism, thus playing a central role in theoretical investigation of strongly correlated systems (Domanski et al., 1996). In its simplest form, the single-band Hubbard model (Hubbard, 1963; Kampf and Schrieffer, 1990; Fano and Ortolani, 1994) reads:

$$
H=-t \sum_{\langle i, j\rangle \sigma}\left(C_{i \sigma}^{+} C_{j \sigma}+H . C\right)+U \sum_{i} n_{i \uparrow} n_{i \downarrow}
$$

Where $C_{i \sigma}^{+} C_{j \sigma}$ and $\mathrm{n}_{\mathrm{i} \sigma}$ are the creation (annihilation) and number operators respectively, for an electron of spin $\sigma$ in the Wannier state on the ith lattice site. $\langle i, j\rangle$ means that only nearest - neighbor site hopping is allowed. H.C. is the Hermitian conjugate and its presence ensures that the dynamical quantities are real. $t$ and $u$ are respectively the hopping and on-site interaction weighting factor. The u term in the Hamiltonian accounts for the dominant part of the Coulomb repulsion.

In this section an attempt is made at describing the method adopted and the reasons for adopting it. The study of strongly correlated electron system is quite a challenge. This is because there are no well controlled analytical techniques to analyze their properties when the Hilbert space increases. The basic results obtained rely heavily on numerical techniques and simulations. The method presented here has been so modified that the improved ground state energy and wave function can be seen at a glance in the $2 \times 2$ step iteration.

The Lanczos algorithm can be viewed as a simplified Arnoldi's algorithm in that it applies to Hermitian matrices. It transforms the original matrix into a tri-diagonal matrix, which is real and symmetric. The basic idea is that a special basis can be constructed where the Hamiltonian has a tri-diagonal representation (Lanczos, 1950; Pettifor and Weaire, 1985; Gulácsi and Kampf, 2008).

As in the standard Lanczos technique, and the modified Lanczos method (Dagotto and Moreo, 1985;

Gagliano et al., 1986), this method requires the selection of an initial trial vector $\left|\phi_{n}\right\rangle$ (normalized to one) where

$\mathrm{n}$ takes values from $0,1,2,3$ and so on. If $H$ acts on $\left|\phi_{n}\right\rangle$ the result can be written as

$$
H\left|\phi_{n}\right\rangle=\frac{\left\langle\phi_{n}|H| \phi_{n}\right\rangle}{\left\langle\phi_{n} \mid \phi_{n}\right\rangle}\left|\phi_{n}\right\rangle+\left|\tilde{\phi}_{n}\right\rangle
$$

Where $\left|\tilde{\phi}_{n}\right\rangle$ is a new state orthogonal to $\left|\phi_{n}\right\rangle$. Since $\left|\phi_{n}\right\rangle$ is normalized, eqn. (8) becomes

$$
\left|\tilde{\phi}_{n}\right\rangle=\left(H\left|\phi_{n}\right\rangle-\left\langle\phi_{n}|H| \phi_{n}\right\rangle\left|\phi_{n}\right\rangle\right) \frac{1}{b_{n}}
$$

The constant $1 / b_{n}$ ensures that $\left|\phi_{n}\right\rangle$ is normalized. 
From eqn.9, we have that $\left\langle\tilde{\phi}_{n} \mid \tilde{\phi}_{n}\right\rangle=\left[\left\langle\phi_{n}\left|H^{2}\right| \phi_{n}\right\rangle-\left(\left\langle\phi_{n}|H| \phi_{n}\right\rangle\right)^{2}\right]{\frac{1}{b_{n}}}^{2}=1$

This gives

$$
\frac{1}{b_{n}}=\left[\left\langle\phi_{n}\left|H^{2}\right| \phi_{n}\right\rangle-\left(\left\langle\phi_{n}|H| \phi_{n}\right\rangle\right)^{2}\right]^{1 / 2}
$$

Now, the action of $\mathrm{H}$ on $\left|\tilde{\phi}_{n}\right\rangle$ gives

$$
H\left|\tilde{\phi}_{n}\right\rangle=\left[H^{2}\left|\phi_{n}\right\rangle-\left\langle\phi_{n}|H| \phi_{n}\right\rangle H\left|\phi_{n}\right\rangle\right] \frac{1}{b_{n}}
$$

So that

Also, it can easily be shown that

$$
c_{n}=\left\langle\tilde{\phi}_{n}|H| \tilde{\phi}_{n}\right\rangle=\left\langle\tilde{\phi}_{n}\left|H^{2}\right| \phi_{n}\right\rangle-\left\langle\phi_{n}|H| \phi_{n}\right\rangle\left\langle\tilde{\phi}_{n}|H| \phi_{n}\right\rangle
$$

If $\left\langle\phi_{0}|H| \phi_{0}\right\rangle$ is denoted by $\in_{0}$ then in the basis $\left|\phi_{n}\right\rangle$ and $\left|\tilde{\phi}_{n}\right\rangle$, a $2 \times 2$ matrix representation of $\mathrm{H}$ is given by

$$
H_{i, j}=\left[\begin{array}{ll}
a & b \\
b & c
\end{array}\right]
$$

This $2 \times 2$ matrix can easily be diagonalized. Its lowest eigenvalue $\epsilon_{n+1}$ is given by

$$
\epsilon_{n+1}=1 / 2\left[\epsilon_{n}+c_{n}-\sqrt{\left(\epsilon_{n}+c_{n}\right)^{2}-4\left(\epsilon_{n} c_{n}-b_{n}^{2}\right)}\right]
$$

Its corresponding eigenvector $\left|\phi_{n}\right\rangle$ is given by

$$
\left|\phi_{n+1}\right\rangle=\alpha|\phi\rangle+\beta|\tilde{\phi}\rangle
$$

Where $\alpha=\sqrt{\frac{b_{n}{ }^{2}}{b_{n}{ }^{2}+\left(\epsilon_{n}-\epsilon_{n+1}\right)^{2}}}$, and $\beta=-\sqrt{\frac{\left(\epsilon_{n}-\epsilon_{n+1}\right)^{2}}{b_{n}{ }^{2}+\left(\epsilon_{n}-\epsilon_{n+1}\right)^{2}}}$

Note that $\alpha$ and $\beta$ are the weights of the improved wave function. $\in_{n+1}$ and $\left|\phi_{n+1}\right\rangle$ are better approximations to $\mathrm{E}_{0}$ (true ground state energy) $\left|\Phi_{0}\right\rangle$ (true ground state wave function) than $\epsilon_{n}$ and $\left|\phi_{n}\right\rangle$ respectively.

The method can be iterated by considering $\left|\phi_{n+1}\right\rangle$ as a new initial trial vector and repeating the steps from eqn. 8 to 17. In each iteration, the orthogonal pairs $\left(\left|\phi_{n}\right\rangle,\left|\tilde{\phi}_{n}\right\rangle\right),\left(\left|\phi_{n+1}\right\rangle,\left|\tilde{\phi}_{n+1}\right\rangle\right)$, etc are normalized.

\section{Application of the Method to a System of Two Electrons on a 3 X 3 Square Lattice}

In this section, the method will be applied to two electrons interacting on a $3 \times 3$ square lattice. The numbers of anti-ferromagnetic states are 81 . Applying the linear combination of states, our new basis states will be

$$
|R\rangle,|S\rangle \text { and }|T\rangle
$$


Where the new basis state $R$ accounts for nine old basis states, $S$ accounts for 36 old basis states and $T$ accounts for 36 old basis states. When the Hubbard Hamiltonian as given in Equation (1), acts on this state, it can be shown that the following will be obtained;

$$
\begin{aligned}
& H|R\rangle=U|R\rangle-2 t|S\rangle \\
& H|S\rangle=-8 t|R\rangle-2 t|S\rangle-4 t|T\rangle \\
& H|T\rangle=-4 t|S\rangle-4 t|T\rangle
\end{aligned}
$$

Choosing our initial trial vector after normalization to be

$$
\left|\phi_{0}\right\rangle=\frac{1}{3}|R\rangle=A_{1}|R\rangle
$$

The first iteration can be carried out

$$
\begin{aligned}
& H\left|\phi_{0}\right\rangle=A_{1} U|R\rangle-2 t A_{1}|S\rangle \\
& \left\langle\phi_{0}|H| \phi_{0}\right\rangle=\epsilon_{0}=U \\
& H\left|\phi_{0}\right\rangle=B_{1}|R\rangle+B_{2}|S\rangle \\
& H^{2}\left|\phi_{0}\right\rangle=\left(B_{1} U-8 t B_{2}\right)|R\rangle+\left(-2 t B_{1}-2 t B_{2}\right)|S\rangle+4 t B_{2}|T\rangle \\
& \left\langle\phi_{0}\left|H^{2}\right| \phi_{0}\right\rangle=9 A_{1}\left(B_{1} U-8 t B_{2}\right) \\
& b_{0}=\left(\left\langle\phi_{0}\left|H^{2}\right| \phi_{0}\right\rangle-\epsilon_{0}^{2}\right)^{1 / 2} \cdot \\
& \left|\tilde{\phi}_{0}\right\rangle=-\frac{1}{b_{0}}\left(B_{1}-\epsilon_{0} A_{1}\right)|R\rangle+\frac{B_{2}}{b_{0}}|S\rangle \\
& \left|\tilde{\phi}_{0}\right\rangle=C_{1}|R\rangle+C_{2}|S\rangle \\
& \mathrm{c}_{0}=0 \\
& \epsilon_{1}=1 / 2\left[\epsilon_{0}+c_{0}-\sqrt{\left(\epsilon_{0}+c_{0}\right)^{2}-4\left(\epsilon_{0} c_{0}-b_{0}^{2}\right)}\right] \\
& \left|\phi_{1}\right\rangle=\alpha_{0}\left|\phi_{0}\right\rangle+\beta_{0}\left|\tilde{\phi}_{0}\right\rangle \\
& \text { where } \alpha_{0}=\sqrt{\frac{b_{0}^{2}}{b_{0}^{2}+\left(\epsilon_{0}-\epsilon_{1}\right)^{2}}} \text { and } \beta_{0}=-\sqrt{\frac{\left(\epsilon_{0}-\epsilon_{1}\right)^{2}}{b_{0}^{2}+\left(\epsilon_{0}-\epsilon_{1}\right)^{2}}}
\end{aligned}
$$

This iteration is arried out five more times before the ground state energy and wave function are obtained. The energy and wave function obtained after an iteration process are called the improved energy and wave function respectively. The improved wave function becomes the trial basis vector for the next iteration.

\section{Application of the Method to a System of Two Electrons on a 5 X 5 Square Lattice}

In this section, the method will be applied to a system of two electrons interacting on a $5 \times 5$ square lattice. The numbers of anti-ferromagnetic states are 625 . Our new basis states based on linear combination of states are arbitrarily chosen to be

$$
|P\rangle,|Q\rangle,|R\rangle,|S\rangle,|T\rangle \text { and }|U\rangle
$$


When the Hubbard Hamiltonian as given in equation (1.2) acts on this state, it can be shown that the following will be obtained;

$$
\begin{aligned}
& H|P\rangle=U|P\rangle-2 t|Q\rangle \\
& H|Q\rangle=-8 t|P\rangle-4 t|R\rangle-2 t|S\rangle \\
& H|R\rangle=-4 t|Q\rangle-2 t|T\rangle \\
& H|S\rangle=-2 t|Q\rangle-2 t|S\rangle-2 t|T\rangle \\
& H|T\rangle=-4 t|R\rangle-4 t|S\rangle-2 t|T\rangle-4 t|U\rangle \\
& H|U\rangle=-2 t|T\rangle-4 t|U\rangle
\end{aligned}
$$

Choosing our initial trial vector to be

$$
\left|\phi_{0}\right\rangle=\frac{1}{5}|P\rangle=A_{1}|P\rangle
$$

The first iteration can be carried out

$$
\begin{aligned}
& H\left|\phi_{0}\right\rangle=A_{1} U|P\rangle-2 t A_{1}|Q\rangle \\
& H\left|\phi_{0}\right\rangle=B_{1}|P\rangle+B_{2}|Q\rangle \\
& \left\langle\phi_{0}|H| \phi_{0}\right\rangle=\epsilon_{0}=U \\
& H^{2}\left|\phi_{0}\right\rangle=\left(B_{1} U-8 t B_{2}\right)|P\rangle-2 t B_{1}|Q\rangle+4 t B_{2}|R\rangle-2 t B_{2}|S\rangle \\
& \left\langle\phi_{0}\left|H^{2}\right| \phi_{0}\right\rangle=25 A_{1}\left(B_{1} U+8 t B_{2}\right) \\
& b_{0}=\left(\left\langle\phi_{0}\left|H^{2}\right| \phi_{0}\right\rangle-\epsilon_{0}^{2}\right)^{1 / 2} \\
& \left|\tilde{\phi}_{0}\right\rangle=\frac{1}{b_{0}}\left(B_{1}-A_{1} \in_{0}\right)|P\rangle+\frac{B_{2}}{b_{0}}|Q\rangle \\
& \left|\tilde{\phi}_{0}\right\rangle=C_{1}|P\rangle+C_{2}|Q\rangle \\
& \mathrm{c}_{0}=0 \\
& \epsilon_{1}=1 / 2\left[\epsilon_{0}+c_{0}-\sqrt{\left(\epsilon_{0}+c_{0}\right)^{2}-4\left(\epsilon_{0} c_{0}-b_{0}^{2}\right)}\right] \\
& \left|\phi_{1}\right\rangle=\alpha_{0}\left|\phi_{0}\right\rangle+\beta_{0}\left|\tilde{\phi}_{0}\right\rangle
\end{aligned}
$$

where $\alpha_{0}=\sqrt{\frac{b_{0}^{2}}{b_{0}^{2}+\left(\epsilon_{0}-\epsilon_{1}\right)^{2}}}$ and $\beta_{0}=-\sqrt{\frac{\left(\epsilon_{0}-\epsilon_{1}\right)^{2}}{b_{0}^{2}+\left(\epsilon_{0}-\epsilon_{1}\right)^{2}}}$

About thirteen more iterations are carried out before the ground state energy and wave function are obtained. Note that after every iteration, the energy and wave function obtained are called the improved energy and wave function. Where

$\left|\phi_{1}\right\rangle=\alpha_{0}\left|\phi_{0}\right\rangle+\beta_{0}\left|\tilde{\phi}_{0}\right\rangle$

is the basis for the next iteration and so on. 


\section{Presentation of Results}

The last column in Tables I and II shows the ground state energies and wave functions for two electrons on a $3 \mathrm{x}$ 3 square lattice respectively. The wave function is presented using the new basis states and this adequately covers all the orthogonal states. The numerical values in the column for the 7th iteration in Table II represent the weights attached to the ground state wave function. It is noted that in the strong coupling regime when $\mathrm{u} / \mathrm{t}$ is positive, the energy is higher than the energy of the weak coupling regime of negative u/t. The last column in Tables III and IV also shows the ground state energies and wave functions for two electrons on a $5 \times 5$ square lattice respectively. The wave function is presented using the new basis states and this adequately covers all the orthogonal states. The numerical values in the column for the 13th iteration in Table IV represent the weights attached to the ground state wave function. It is noted that in the strong coupling regime when $\mathrm{u} / \mathrm{t}$ is positive, the energy is higher than the energy of the weak coupling regime of negative $\mathrm{u} / \mathrm{t}$.

Table I: Ground state energy ( $E / t)$ for two electrons on a 3 × 3 square lattice as a function $u / t(a t t=1)$.

\begin{tabular}{|l|l|l|l|l|l|l|l|}
\hline $\mathrm{u} / \mathrm{t}$ & $\epsilon_{1}$ & $\epsilon_{2}$ & $\epsilon_{3}$ & $\epsilon_{4}$ & $\epsilon_{5}$ & $\epsilon_{6}$ & $\epsilon_{7}$ \\
\hline 5.00 & -3.8151 & -7.5512 & -7.6493 & -7.6493 & -7.6493 & -7.6493 & -7.6493 \\
\hline 3.00 & -4.2170 & -7.6088 & -7.7493 & -7.7522 & -7.7522 & -7.7522 & -7.7522 \\
\hline 1.00 & -4.7720 & -7.6963 & -7.8929 & -7.8999 & -7.9000 & -7.9000 & -7.9000 \\
\hline 0.00 & -5.1231 & -7.7640 & -7.9889 & -7.9995 & -7.9999 & -8.0000 & -8.0000 \\
\hline-1.00 & -5.5311 & -7.8605 & -8.1083 & -8.1237 & -8.1247 & -8.1247 & -8.1247 \\
\hline-3.00 & -6.5311 & -8.1997 & -8.4558 & -8.4819 & -8.4845 & -8.4848 & -8.4848 \\
\hline-5.00 & -7.7720 & -8.8437 & -9.0432 & -9.0719 & -9.0759 & -9.0765 & -9.0766 \\
\hline
\end{tabular}

Table II: Ground state wave function $\left(\Psi_{\mathrm{G}}\right)$ for two electrons on a $3 \times 3$ square lattice at $u / t=-5.00$ to 5.00 .

\begin{tabular}{|c|c|c|c|c|c|}
\hline \multirow[t]{2}{*}{ INTERACTION STRENGTH $\quad u / t$} & \multirow{2}{*}{$\begin{array}{c}\text { WAVE } \\
\text { FUNCTIONS }\end{array}$} & \multicolumn{4}{|c|}{ WEIGHT OF WAVE FUNCTIONS } \\
\hline & & $1^{\mathrm{ST}}$ & $3^{\mathrm{RD}}$ & $5^{\mathrm{TH}}$ & $7^{\mathrm{TH}}$ \\
\hline \multirow[t]{3}{*}{5.00} & $\left|1^{\prime}\right\rangle$ & 0.1377 & 0.0711 & 0.0695 & 0.0695 \\
\hline & $\left|2^{\prime}\right\rangle$ & 0.1518 & 0.1112 & 0.1099 & 0.1099 \\
\hline & $\left|3^{\prime}\right\rangle$ & & 0.1189 & 0.1204 & 0.1204 \\
\hline \multirow[t]{3}{*}{3.00} & $\left|1^{\prime}\right\rangle$ & 0.1616 & 0.0852 & 0.0823 & 0.0822 \\
\hline & $\left|2^{\prime}\right\rangle$ & 0.1458 & 0.1125 & 0.1105 & 0.1105 \\
\hline & $\left|3^{\prime}\right\rangle$ & & 0.1154 & 0.1178 & 0.1178 \\
\hline \multirow[t]{3}{*}{1.00} & $\left|1^{\prime}\right\rangle$ & 0.1899 & 0.1054 & 0.1000 & 0.0998 \\
\hline & $\left|2^{\prime}\right\rangle$ & 0.1370 & 0.1137 & 0.1111 & 0.1110 \\
\hline & $\left|3^{\prime}\right\rangle$ & & 0.1099 & 0.1137 & 0.1139 \\
\hline \multirow[t]{3}{*}{0.00} & $\left|1^{\prime}\right\rangle$ & 0.2051 & 0.1187 & 0.1115 & 0.1111 \\
\hline & $\left|2^{\prime}\right\rangle$ & 0.1314 & 0.1142 & 0.1113 & 0.1111 \\
\hline & $\left|3^{\prime}\right\rangle$ & & 0.1060 & 0.1109 & 0.1111 \\
\hline \multirow[t]{3}{*}{-1.00} & $\left|1^{\prime}\right\rangle$ & 0.2266 & 0.1345 & 0.1252 & 0.1248 \\
\hline & $\left|2^{\prime}\right\rangle$ & 0.1250 & 0.1142 & 0.1112 & 0.1109 \\
\hline & $\left|3^{\prime}\right\rangle$ & & 0.1011 & 0.1072 & 0.1076 \\
\hline-3.00 & $\left|1^{\prime}\right\rangle$ & 0.2499 & 0.1739 & 0.1608 & 0.1599 \\
\hline
\end{tabular}


Table II Continues

\begin{tabular}{|l|l|l|l|l|l|}
\hline & $\left|2^{\prime}\right\rangle$ & 0.1103 & 0.1119 & 0.1095 & 0.1091 \\
\hline & $\left|3^{\prime}\right\rangle$ & & 0.0878 & 0.0965 & 0.0974 \\
\hline-5.00 & $\left|1^{\prime}\right\rangle$ & 0.2740 & 0.2186 & 0.2057 & 0.2044 \\
\hline & $\left|2^{\prime}\right\rangle$ & 0.0949 & 0.1044 & 0.1038 & 0.1034 \\
\hline & $\left|3^{\prime}\right\rangle$ & & 0.0702 & 0.0801 & 0.0815 \\
\hline
\end{tabular}

Table III: Ground state energy of the Hubbard model with two electrons on a 5 x 5 square lattice as a function of $u / t(a t t=1)$. Results are presented for all iterations.

\begin{tabular}{|c|c|c|c|c|c|c|}
\hline $\begin{array}{c}\text { INTERACTION } \\
\text { STRENGTH } \mathbf{u} / \mathbf{t}\end{array}$ & \multicolumn{7}{|c|}{ ENERGY $(\mathbf{u} / \mathbf{t})$} \\
\hline & \multicolumn{7}{|c|}{ ITERATIONS } \\
\hline & $1^{\mathrm{ST}}$ & $4^{\mathrm{TH}}$ & $7^{\mathrm{TH}}$ & $10^{\mathrm{TH}}$ & $13 \mathrm{TH}$ & $14^{\mathrm{TH}}$ \\
\hline & -2.2170 & -7.1677 & -7.8090 & -7.8802 & -7.8870 & -7.8873 \\
\hline 5.00 & -2.7720 & -7.2146 & -7.8441 & -7.9114 & -7.9175 & -7.9178 \\
\hline 3.00 & -3.5311 & -7.2760 & -7.8890 & -7.9582 & -7.9647 & -7.9650 \\
\hline 1.00 & -4.0000 & -7.3208 & -7.9193 & -7.9919 & -7.9992 & -7.9996 \\
\hline 0.00 & -4.5311 & -7.3879 & -7.9599 & -8.0374 & -8.0460 & -8.0466 \\
\hline-1.00 & -5.7720 & -7.6706 & -8.1159 & -8.1979 & -8.2106 & -8.2116 \\
\hline-3.00 & -7.2170 & -8.3215 & -8.5490 & -8.5992 & -8.6093 & -8.6103 \\
\hline-5.00 & & & & & & \\
\hline
\end{tabular}

Table IV: Ground state wave function $\left(\Psi_{\mathrm{G}}\right)$ for two electrons on a $5 \times 5$ square lattice at $\mathrm{u} / \mathrm{t}=\mathbf{- 5 . 0 0}$ to $\mathbf{5 . 0 0}$.

\begin{tabular}{|c|l|l|l|l|l|l|}
\hline \multirow{2}{*}{$\mathbf{u} / \mathbf{t}$} & $\begin{array}{c}\text { WAVE } \\
\text { FUNCTION }\end{array}$ & \multicolumn{5}{|c|}{ WEIGHTS OF WAVE FUNCTION } \\
\hline & & $\mathbf{1}^{\text {ST }}$ & $\mathbf{4}^{\text {TH }}$ & $7^{\text {TH }}$ & $\mathbf{1 0}^{\text {TH }}$ & $13^{\text {TH }}$ \\
\hline 5.00 & $\left|1^{\prime}\right\rangle$ & 0.0970 & 0.0525 & 0.0239 & 0.0255 & 0.0225 \\
\hline & $\left|2^{\prime}\right\rangle$ & 0.0875 & 0.0541 & 0.0451 & 0.0380 & 0.0369 \\
\hline & $\left|3^{\prime}\right\rangle$ & & 0.0541 & 0.0428 & 0.0412 & 0.0398 \\
\hline & $\left|4^{\prime}\right\rangle$ & & 0.0413 & 0.0421 & 0.0416 & 0.0409 \\
\hline & $\left|5^{\prime}\right\rangle$ & & 0.0287 & 0.0397 & 0.0411 & 0.0417 \\
\hline & $\left|6^{\prime}\right\rangle$ & & 0.0104 & 0.0326 & 0.0399 & 0.0421 \\
\hline 3.00 & $\left|1^{\prime}\right\rangle$ & 0.1139 & 0.0610 & 0.0313 & 0.0305 & 0.0276 \\
\hline & $\left|2^{\prime}\right\rangle$ & 0.0822 & 0.0540 & 0.0463 & 0.0390 & 0.0380 \\
\hline & $\left|3^{\prime}\right\rangle$ & & 0.0537 & 0.0425 & 0.0413 & 0.0400 \\
\hline & $\left|4^{\prime}\right\rangle$ & & 0.0407 & 0.0415 & 0.0413 & 0.0407 \\
\hline & $\left|5^{\prime}\right\rangle$ & & 0.0274 & 0.0391 & 0.0406 & 0.0412 \\
\hline & $\left|6^{\prime}\right\rangle$ & & 0.0106 & 0.0320 & 0.0393 & 0.0413 \\
\hline 1.00 & $\left|1^{\prime}\right\rangle$ & 0.1324 & 0.0738 & 0.0422 & 0.0387 & 0.0354 \\
\hline & $\left|2^{\prime}\right\rangle$ & 0.0750 & 0.0546 & 0.0483 & 0.0407 & 0.0398 \\
\hline & $\left|3^{\prime}\right\rangle$ & & 0.0524 & 0.0421 & 0.0416 & 0.0402 \\
\hline & $\left|4^{\prime}\right\rangle$ & & 0.0392 & 0.0405 & 0.0409 & 0.0403 \\
\hline & $\left|5^{\prime}\right\rangle$ & & 0.0252 & 0.0379 & 0.0396 & 0.0404 \\
\hline
\end{tabular}




\begin{tabular}{|l|l|l|l|l|l|l|}
\hline & $\left|6^{\prime}\right\rangle$ & & 0.0103 & 0.0305 & 0.0380 & 0.0400 \\
\hline 0.00 & $\left|1^{\prime}\right\rangle$ & 0.1414 & 0.0828 & 0.0500 & 0.0447 & 0.0410 \\
\hline & $\left|2^{\prime}\right\rangle$ & 0.0707 & 0.0550 & 0.0497 & 0.0419 & 0.0410 \\
\hline & $\left|3^{\prime}\right\rangle$ & & 0.0511 & 0.0419 & 0.0417 & 0.0402 \\
\hline & $\left|4^{\prime}\right\rangle$ & & 0.0378 & 0.0398 & 0.0406 & 0.0400 \\
\hline & $\left|5^{\prime}\right\rangle$ & & 0.0236 & 0.0369 & 0.0388 & 0.0397 \\
\hline & $\left|6^{\prime}\right\rangle$ & & 0.0098 & 0.0292 & 0.0368 & 0.0390 \\
\hline-1.00 & $\left|1^{\prime}\right\rangle$ & 0.1499 & 0.0939 & 0.0599 & 0.0528 & 0.0485 \\
\hline & $\left|2^{\prime}\right\rangle$ & 0.0662 & 0.0554 & 0.0513 & 0.0435 & 0.0426 \\
\hline & $\left|3^{\prime}\right\rangle$ & & 0.0491 & 0.0414 & 0.0417 & 0.0403 \\
\hline & $\left|4^{\prime}\right\rangle$ & & 0.0359 & 0.0387 & 0.0400 & 0.0396 \\
\hline & $\left|5^{\prime}\right\rangle$ & & 0.0217 & 0.0354 & 0.0376 & 0.0388 \\
\hline & $\left|6^{\prime}\right\rangle$ & & 0.0092 & 0.0275 & 0.0352 & 0.0375 \\
\hline-3.00 & $\left|1^{\prime}\right\rangle$ & 0.1644 & 0.1209 & 0.0890 & 0.0788 & 0.0728 \\
\hline & $\left|2^{\prime}\right\rangle$ & 0.0570 & 0.0549 & 0.0546 & 0.0477 & 0.0470 \\
\hline & $\left|3^{\prime}\right\rangle$ & & 0.0425 & 0.0389 & 0.0409 & 0.0396 \\
\hline & $\left|4^{\prime}\right\rangle$ & & 0.0301 & 0.0347 & 0.0374 & 0.0374 \\
\hline & $\left|5^{\prime}\right\rangle$ & & 0.0169 & 0.0303 & 0.0334 & 0.0351 \\
\hline & $\left|6^{\prime}\right\rangle$ & & 0.0072 & 0.0221 & 0.0297 & 0.0323 \\
\hline-5.00 & $\left|1^{\prime}\right\rangle$ & 0.1749 & 0.1680 & 0.1520 & 0.1191 & 0.1139 \\
\hline & $\left|2^{\prime}\right\rangle$ & 0.0485 & 0.0466 & 0.0574 & 0.0508 & 0.0509 \\
\hline & $\left|3^{\prime}\right\rangle$ & & 0.0250 & 0.0233 & 0.0360 & 0.0356 \\
\hline & $\left|4^{\prime}\right\rangle$ & & 0.0125 & 0.0147 & 0.0306 & 0.0311 \\
\hline & $\left|5^{\prime}\right\rangle$ & & & 0.0092 & 0.0249 & 0.0268 \\
\hline & $\left|6^{\prime}\right\rangle$ & & & & 0.0202 & 0.0223 \\
\hline & & & & & \\
\hline & & & & & &
\end{tabular}

\section{DISCUSSION OF RESULTS}

The results presented in the tables above for the ground state energy and the ground state wave function was obtained using Eqns. (8) and (9) respectively. From results presented, both on-site and inter-site cases with respect to separation distances were carefully considered and there are strong evidences of eigen functions corresponding to non-zero eigen values that are $\mathrm{u}$-independent. The presence of such eigen state is important for the following reasons.

First of all, several authors observed (Galan and Verges 1991, Metzner and Vollhardt 1989) that weak coupling expansions often provide a good approximation for the Hubbard model at intermediate coupling. A possible explication for this is the emergence of a huge number of eigen states in the spectrum of the Hubbard model with non-zero interaction, which are present in the non-interacting case as well in the spectrum of the model. A second aspect which must be mentioned here is that the eigen states related to zero eigen value are in fact many-body eigen states of the kinetic energy term with no double occupancy. Such states apparently totally avoid double occupancy at no cost of energy, consequently are important in the study of the pairing mechanism in the Hubbard model (Cini et al., 2001; Cini and Stefanucci, 2001; Perfetto and Cini, 2004), and as seen from the presented results, easily emerge in the spectrum.

At this point, we underline that contrary to the practice used in the theoretical studies up to this moment (Balzarotti et al., 2004), double occupancy avoiding eigen states emerge not only at zero energy, but also at much lower energy values, closely situated to the ground state energy. We exemplify this statement by the eigen state close to zero and zero interaction strength corresponding to energy -8 (in t units). These eigen states being $u$ independent, their energy remain unchanged if the strength of the on-site interaction is increased, while the 
ground-state energy increases if $u$ is increased. Consequently, starting from these states, the contribution in the pairing mechanism of the kinetic energy eigen states not containing double occupancy could be much more efficiently taken into account than considered up to today.

\section{CONCLUSION}

In this paper a modification of the standard Lanczos technique has been analyzed for two electrons on twodimensional lattices and the results obtained compare well with previously obtained using other techniques. This approach has been successfully applied to one- dimensional lattice. Regarding the specific physical problem analyzed in this paper, that is the ground state energy of $3 \times 3$ and $5 \times 5$ lattices, it can be safely concluded that there is no evidence of an anomalous behavior in the iterations.

\section{REFERENCES}

Balzarotti A, et. al. (2004). cond-mat/0411101.

Cini M, Perfetto E and Steffanucci G (2001). Eur. Phys. Jour. B20, 91.

Cini M and Stefanucci G (2001). Jour. of Phys. C13, 1279.

Dagotto E and Moreo A (1985). Improved Hamiltonian Variational Technique for Lattice Models. Phys. Rev. D31(1): 865-870.

Dagotto E (1994). Correlated Electrons in High-Temperature Superconductors. Rev. Mod. Phys. 66(3): pp763840.

Domanski T and Wysokiński KI (1999). Superconducting Phases in the Presence of Coulomb Interaction: from Weak to Strong Correlations. Phys. Rev. B 59(1): pp173-176.

Enaibe E (2004). PhD Thesis, (unpublished) "a Variational Approach to the Study of Highly Correlated Electron Systems" University of Benin.

Fano G and Ortolani F (1994). Hubbard Model with Unconstrained Hopping on a Finite Number of Sites. Phys. Rev. B. 49(11): pp7205-7209.

Gagliano ER, Dagotto E, Moreo A and Alcaraz F (1986). Correlation Functions of the Antiferromagnetic Heisenberg Model using a Modified Lanczos Method. Phys. Rev. B 34(3): pp1677-1682.

Gulácsi Z, Kampf A and Vollhardt D (2008). Exact Many Electron Ground states on Diamond and Triangle Hubbard Chains. Prog. Theor. Phys. Supplement No. 176, pp1-21.

Galan J and Verges J (1991). Phys. Rev. B44, 10093.

Hubbard J (1963). Electron Correlations in Narrow Band. Proc. Roy. Soc. London A 276, pp238-257.

Lieb EH (1994). In Proceedings of the Xlth International Conference of Mathematical Physics, Paris. Edited by D Lagolnitzer (International Press, Paris. 1995). Pp 392.

Kampf AP and Schrieffer JR (1990). Spectral Function and Photoemission Spectra in Antiferromagnetically Correlated Metals. Phys. Rev. B 42(13): pp7967-7974.

Lanczos C (1950). An Iteration Method for the Solution of the Eigen value Problem of Linear Differential and Integral Operators J. Res. Nat. Bur. Stand. 45: pp 255-282.

Perfetto E and Cini M (2004). Jour. of Phys. C16, 4845.

Pettifor A and Weaire W (1985). Recursion Method and its Applications. Springer, Solid State Sciences Ser. USA.

Vollhardt D, Blumer N, Held K, Koller M, Schlipf J and Ulmke M (1997). Non Perturbative Approaches to Magnetism in Strongly Correlated Electron Systems. Physik. B 103(2): pp283-292. 